\title{
Development of a Dashboard for Rare Diseases - A Technical Case Report
}

\author{
Liz A. LEUTNER ${ }^{\text {a,1 }}$, Franziska BATHELT ${ }^{\text {a }}$, Brita SEDLMAYR , \\ Martin SEDLMAYR ${ }^{\mathrm{a}}$ and Michele $\mathrm{ZOCH}^{\mathrm{a}}$ \\ anstitute for Medical Informatics and Biometry, Carl Gustav Carus Faculty of \\ Medicine, Technische Universität, Dresden, Germany
}

\begin{abstract}
About 30 million people suffer from a rare disease in Europe. Those affected face a variety of problems. These include the lack of information and difficult access to scientific knowledge for physicians. For a higher visibility of rare diseases and high-quality research, effective documentation and use of data are essential. The aim of this work is to optimize the processing, use and accessibility of data on rare diseases and thus increase the added value from existing information. While dashboards are already being used to visualize clinical data, it is unclear what requirements are prevalent for rare diseases and how these can be implemented with available development tools so that a highly accepted dashboard can be designed. For this purpose, based on an analysis of the current situation and a requirements analysis, a prototype dashboard for the visualization of up-to-date key figures on rare diseases was developed at the University Hospital Carl Gustav Carus in Dresden. The development was based on the user-centered design process in order to achieve a high-level user-friendliness. The requirements analysis identified parameters that stakeholders wanted to see, focusing primarily on statistical analyses. The dashboard handles the automated calculation of statistics as well as their preparation and provision. The evaluations showed the prototypical dashboard would be considered valuable and used by potential users. This work demonstrates that stakeholders are interested in access to prepared information and exemplifies a way to implement it. The dashboard can increase the usage of existing information in terms of a higher accessibility and thus improve the knowledge about rare diseases.
\end{abstract}

Keywords. Rare Diseases, Requirements Analysis, User-Centered Design, Dashboard

\section{Introduction}

\subsection{Background}

In Europe, a disease is considered rare at a prevalence of 50 per 100,000 people [7]. To date, between 6,000 and 8,000 rare diseases are known. Genetic causes underlie a large proportion of these [10]. The exact number of people affected cannot be determined due to the lack of epidemiological data on these diseases [8]. It is estimated that 30 million people in Europe suffer from a rare disease. Thus, despite their small numbers in each case, they represent a major challenge in their entirety, especially since these diseases

${ }^{1}$ Corresponding Author, Liz A. Leutner, Institute for Medical Informatics and Biometry, Carl Gustav Carus Faculty of Medicine, Technische Universität Dresden, Dresden, Germany; E-mail: liz_annika.leutner@tu-dresden.de. 
are often life-threatening and result in a massive loss of quality of life for the patients [3]. Added to this it often takes many years before affected patients receive a correct diagnosis. Before that, they receive many misdiagnoses, which sometimes lead to unnecessary interventions with side effects [1]. Also, scientific knowledge is lacking [8] and access to appropriate care is often difficult [3].

The core cause of these hurdles are the small numbers of cases for each disease, makes appropriate documentation and use of them to generate new reliable knowledge urgent. For example, a study of the prevalence of rare diseases by the European Organization for Rare Diseases (Eurodis) and Orphanet found that reliable data are often lacking, there is little consistency between different sources of information, and epidemiological studies are often of poor methodological quality [9]. In addition, rare diseases can only be inadequately coded using the ICD-10 (International Classification of Diseases, 10th edition). It contains only about 500 rare diseases, of which about 240 are assigned to a specific ICD code [1]. Therefore, Orphanet provides a database with an index of rare diseases based on specialized publications. All rare diseases in this database are assigned a specific identifier called Orpha code or Orpha number. These identification numbers are arranged in a polyhierarchical classification system and are intended to facilitate data collection, research, and analysis related to rare diseases [10].

At University Hospital Carl Gustav Carus in Dresden (UKD), a parameterized form for recording rare diseases is used. This form was developed by the University Center for Rare Diseases (German: "UniversitätsCentrum für Seltene Erkrankungen" 2; USE) [6] with support from the Business Unit for Information Technology. With this form, rare diseases can be identified by its Orpha codes, ICD codes and associated proteins and genes. The Orpha catalog serves as the basis for this. This form is embedded in the hospital information system (Dedalus ORBIS) and has been mandatory to fill out since 2017 (specifying "No Rare Diseases" is also possible). The data collected through this is currently being manually analyzed by the USE non-medical coordinator.

\subsection{Objective and Requirements}

The aim of this work is to provide an automated calculation and visualization of data on rare diseases in accordance to the needs of the USE members. This should improve the visibility of rare diseases and ultimately the situation of the affected patients with regard to raising awareness of the "orphans" of medicine. For this purpose, a prototype dashboard is created, which provides up-to-date information on data collected on rare diseases. The dashboard visualizes the data and allows a flexible adaption of the provided information according to the respective interests. The development is done in a userfriendly process to ensure acceptance and future use by clinical staff.

\section{State of the art}

\subsection{Related Work}

The dashboard is based on patient data, specifically on diagnoses of rare diseases. A literature search of German and English research articles (without limitation of publication date) on topic of rare disease dashboards revealed that there has been little

\footnotetext{
${ }^{2}$ https://www.uniklinikum-dresden.de/de/das-klinikum/universitaetscentren/use/
} 
work on visualizing clinical data specifically for rare diseases. All papers focusing on rare diseases in general were included. We excluded papers that focused either on pharmacy or on specific individual disease patterns or clinical trials. Table 1 shows the databases and number of results before and after a title-abstract-screening by using the following search string: Dashboard AND ("Rare Diseases" OR "Rare Disease" OR "Orphan Diseases" OR "Orphan Disease"). As a result, none of the papers found could be included.

Table 1. Literature research: Database and results

\begin{tabular}{lll}
\hline Databases & Total Results & Results after Title-Abstract-Screening \\
\hline PubMed & 4 & 0 \\
IEEE & 0 & 0 \\
Web of Science & 3 & 0 \\
\hline
\end{tabular}

We were not able to include a paper, but a closer look at dashboards for clinical data in general revealed recent studies showing that visualization of clinical data in the form of dashboards have high added value. In the systematic review by KHAIRAT ET AL., it is described that visualization within dashboards can, inter alia, reduce the time required for data acquisition and data processing as well as improve the situation awareness and cognitive processing of data [5].

\subsection{Short comings}

Although there are papers describing the use and potential of dashboards in clinical practice, we could not identify any papers on dashboards for rare diseases in general that provide information on what requirements dashboards need to fulfil in the context of rare diseases, which clinical parameters are relevant and how these can be displayed by automated visualization

\section{Concept}

For the entire process of planning and creating the dashboard, a user-centered design (UCD) was chosen that puts the intended users at the center of the development activities. Overall, this process can be divided into the following steps: (1) Analysis of the current situation, (2) requirements analysis, (3) mockup and dashboard design, (4) development of the dashboard and evaluation. The analyses in advance provide information about the usefulness, the desirability, and specific requirements of the dashboard. This ensures the acceptance of the dashboard and the identification of extension proposals.

(1) In the first step, an analysis of the current situation was performed. A precise state analysis regarding the collection and use of data on rare diseases is required to be able to integrate the dashboard well into the existing processes at the UKD and provides the minimum requirements for the necessary calculations that are currently carried out manually. For this purpose, an expert interview was performed with the person currently primarily responsible for evaluating the data collected on rare diseases. In detail, the asis analysis should provide information about existing data, its collection and processing as well as information about who uses this data for what purpose. 
(2) In the next step, a requirements analysis with representatives of potential user groups was carried out using a questionnaire. This should provide information about the usefulness, the motivation of the usage as well as specifications of the dashboard. The aim was to ask whether and for what reasons the users would use a dashboard of this information and what wishes they have regarding the content and form of the dashboard.

(3) Based on the previous analyses, a first draft of the user interface in the form of mock-ups was designed and presented to potential users. This was to check whether the expectations of potential users are being met and where there is still room for improvement.

(4) After specifying the user interface and functions, the dashboard was created and was tested again with users in the context of another workshop.

\section{Implementation}

\subsection{Solution / Results}

\subsubsection{Analysis of the current situation}

The diagnoses of rare diseases are recorded at the UKD using a parameterized form. The expert interview revealed, that this data is collected and evaluated manually about once a year by the non-medical coordinator of the USE. The calculations currently carried out include, for example, the proportion of cases/patients with a documented rare disease in the total number of cases/patients, the absolute number of cases/patients with at least one entry for a rare disease, the number of cases/patients per number of different diagnosed rare diseases, the ten most frequently recorded rare diseases or the number of cases and patients with a documented rare disease per department. The calculated key figures are made available to the Executive Board, where they are used for resource calculation. The non-medical coordinator reported the rapid loss of comprehensibility due to the processing of data in various manually created Excel tables. This shows the high manual effort, which is time-consuming as well as prone to error, which can easily be reduced by IT-supported, automated visualizations. The errors relate to the calculation of key figures and has no direct impact on patients or their health care

\subsubsection{Requirements analysis}

For the collection of the requirements, a self-developed questionnaire was used, which comprised four parts: (1) reason for use, (2) content design, (3) display of results as well as (4) meaningfulness and visualization of data.

(1) Reasons for use: Given the possibility of accessing statistics on key figures on rare diseases, the respondents would use them mainly for research purposes and out of professional interest. In second place would be their use for resource planning.

(2) Content design: In addition to the statistics already calculated (see 4.1.1), statistics on the following topics (as expansion options) are also important to participants:

- The methods of analysis of the diagnostic

- The information about whether the diagnostic status is genetically confirmed, laboratory chemical or clinical

- The time duration of the diagnostic process

- The disciplines actively involved in the care 
- The cost of diagnostics and therapies

- The testing of family members

- The risk to family members of developing the same rare disease as the patient

- The effect of the diagnosis on the patient

- The time spent in the USE, including all boards

- The ratio of specific ICD-10 codes with and without a rare disease indication

- The division of the patient cohort into under and over 18 years old

- The presentation of information on individual patients.

With regard to the general design of the content, the participants also wish diagrams on ratios and yearly comparisons with the possibility of reducing these to months. Furthermore, information about the data source is considered mandatory.

(3) Display of results: Participants would like to be able to flexibly decide which statistics are displayed. In addition, the majority of them state that graphics and visualizations of figures help them to get a quick overview. There are heterogeneous opinions as to whether they prefer a clear, simpler presentation. Furthermore, they mention the following design aspects as important:

- The division of information packages into different tabs

- Expandable information instead of its direct complete presentation

- The possibility of corrections

- The possibility of including and excluding diagnoses and patients

- The possibility to filter by time periods

(4) Meaningfulness of the evaluation and visualization of key figures on rare diseases: The majority of the participants (4/5) consider the evaluation of the collected data on rare diseases to be important and consider a clear presentation of the data in the form of a dashboard to be useful and desirable. They state that access to the relevant statistics would represent additional value for them and that they trust statistics.

\subsubsection{Development of the Dashboard}

Based on the requirements analysis, mockups were created using the tool Balsamiq ${ }^{3}$ for the design of the user interface. These were presented to potential users in form of a walkthrough at a workshop. As a result of this workshop, all participants expressed a high level of acceptance of the designs. A small suggestion for improvement from the participants related to the language selection was taken into account in the next iteration. The dashboard was then designed using Dashbuilder ${ }^{4}$ with data of a locally created test database. This tool allows the intuitive creation of dashboards as a web application and is free of charge. The dashboard takes the required data directly from a specified database so that the information provided is up-to-date. This database contains the information corresponding to the data collected by the form for recording rare diseases at the UKD.

The dashboard automates all calculations that were previously carried out manually. These statistics are displayed graphically on various pages. There is a subdivision into "general occurrence of rare diseases" and "occurrence of specific rare diseases". On both sides, the user can decide whether the calculations should be carried out on the basis of the number of patients or cases. This corresponds to the previous distinction in manual calculation. Using various filters (e.g. period, specialist department or a specific rare disease), users can adjust the information presented to them (see Figure 1).

\footnotetext{
${ }^{3}$ https://balsamiq.com/

${ }^{4}$ http://dashbuilder.org/
} 


\subsection{System in Use}

The prototype was tested based on the local database using synthetic data. The limitation to the smaller data set enables manual quality assurance. A further workshop with stakeholders (physician and non-medical coordinator of a rare diseases center) revealed that this prototype was evaluated as useful for their own work and that the visualization of the required parameters was considered helpful. The dashboard is only intended to be access by authorized clinicians who have access to the data anyway.

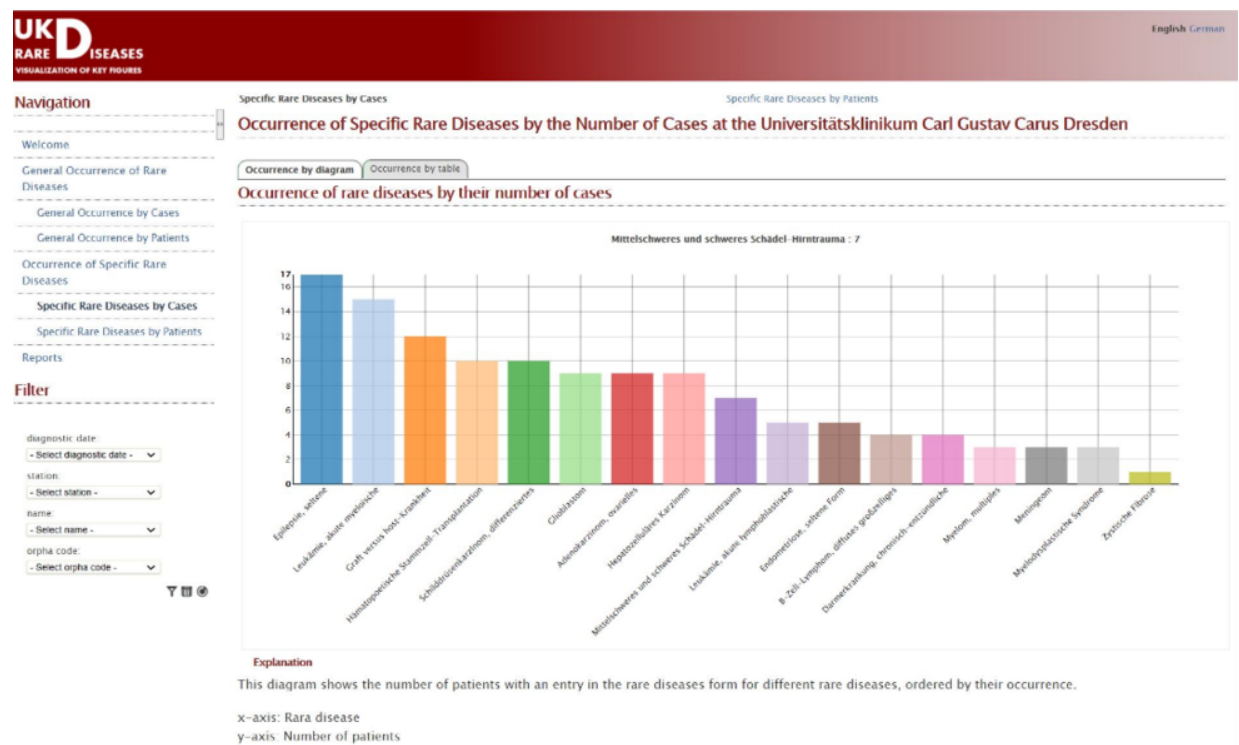

Figure 1. Screenshot of the page "General Occurrence of Rare Diseases by the Number of Cases at the Universitätsklinikum Carl Gustav Carus Dresden” based on synthetic data

\section{Lessons learned}

A prototypical dashboard was created according to the user's wishes, which can be integrated into the existing processes based on the analysis of the current situation. Its acceptance was confirmed and expansion options were identified (see 4.1.2).

The constant exchange with users - as it is recommended by the method of usercentered design - has paid off in terms of usability and integration of the dashboard. Nevertheless, it is currently being investigated whether the dashboard will be delivered in the form of the prototype. Careful selection of the tool according to the requirements facilitates the creation of the dashboard. In retrospect, the tool Superset ${ }^{5}$ would have been a good option too, which is at least as intuitive to use as Dashbuilder and free of charge as well. Superset is already used for a dashboard for forecasting bed occupancy in the context of the COVID-19 pandemic at the UKD. In the long term, the dashboard for rare diseases will also be transferred to Superset in the sense of a uniform tool [11].

\footnotetext{
${ }^{5}$ https://superset.apache.org/
} 
The developed dashboard cannot be clearly categorized as a "quality dashboard" or a "clinical dashboard" [2] because quantitative indicators are calculated and neither quality indicators are determined nor individual patient data are presented. Nevertheless, during development, general guidelines for healthcare dashboard design were considered, such as easy-to-read, graphical displays and system connectivity and integration [4,14]. The provided dashboard differs in terms of its absence of focus on individual patients and their treatments, compared to more common conditions that do not belong to the group of rare diseases (comparable examples [12,13]). This supports the statement by KHARATI ET AL. regarding the dependence of dashboard design on medical context. Nevertheless, the dashboard could be expanded to that effect, which was also one of the further suggestions made by the users.

In general, the dashboard can also be used at other clinics, as it is based on opensource software. The prerequisite for this would also be the documentation by means of Orpha codes. Instructions for implementation of the form developed by the USE will be provided in a future technical case report. Consequently, we will also check the data quality, as the quality of the information presented in the dashboard depends directly on the source data of the form.

\section{Conclusion}

A prototype dashboard was implemented that automates previously manually performed calculations and graphically displays the resulting statistics according to the user's wishes. The entire process was based on user-centered design, so that there was a constant exchange with potential users and the product is intuitive to use. Overall, it remains to be stated:

- The implementation of an automated calculation of metrics on rare diseases is relatively straightforward due to existing software.

- A prototypical exemplary dashboard was created using user centered design.

- Potential users of a dashboard from different user groups consider access to such a dashboard useful.

- Opportunities to expand the dashboard (e.g. new information) were identified.

By calculating and visualizing numbers of diagnoses, patients and cases, the masses of different rare diseases are made quantifiable. By evaluation on the basis of Orpha coding, clinical pictures are also recorded and made visible, which cannot be documented with ICD-10 and thus cannot be evaluated. This goes hand in hand with the users' research intent: to increase access to data and visibility of rare diseases.

\section{Declarations}

Conflict of Interest: The authors declare that there is no conflict of interest.

Author contributions: LAL, MZ: conception of the work; LAL: first draft of the manuscript (on basis of bachelor thesis); MZ: substantial revision of the manuscript. All authors contributed substantial ideas and participated in editing and revising of the manuscript. All authors approved the manuscript in the submitted version and take 
responsibility for the scientific integrity of the work.

Acknowledgement: The authors would like to thank the University Center for Rare Diseases Dresden for their support.

\section{References}

[1] S. Blöß, C. Klemann, A.-K. Rother, S. Mehmecke, U. Schumacher, U. Mücke, M. Mücke, C. Stieber, F. Klawonn, X. Kortum, W. Lechner, and L. Grigull, Diagnostic needs for rare diseases and shared prediagnostic phenomena: Results of a German-wide expert Delphi survey, PLOS ONE. 12 (2017). doi:10.1371/journal.pone.0172532.

[2] D. Dowding, R. Randell, P. Gardner, G. Fitzpatrick, P. Dykes, J. Favela, S. Hamer, Z. WhitewoodMoores, N. Hardiker, E. Borycki, and L. Currie, Dashboards for improving patient care: Review of the literature, Int. J. Med. Inf. 84 (2015) 87-100. doi:10.1016/j.ijmedinf.2014.10.001.

[3] N. Guffon, B. Heron, B. Chabrol, F. Feillet, V. Montauban, and V. Valayannopoulos, Diagnosis, quality of life, and treatment of patients with Hunter syndrome in the French healthcare system: a retrospective observational study, Orphanet J. Rare Dis. 10 (2015) 43. doi:10.1186/s13023-015-02590 .

[4] M. Karami, M. Langarizadeh, and M. Fatehi, Evaluation of Effective Dashboards: Key Concepts and Criteria, Open Med. Inform. J. 11 (2017) 52-57. doi:10.2174/1874431101711010052.

[5] S.S. Khairat, A. Dukkipati, H.A. Lauria, T. Bice, D. Travers, and S.S. Carson, The Impact of Visualization Dashboards on Quality of Care and Clinician Satisfaction: Integrative Literature Review, JMIR Hum. Factors. 5 (2018). doi:10.2196/humanfactors.9328.

[6] T. Kretschmer, Implementierung einer KIS-basierten Anwendung zur Erhebung seltener Erkrankungen am Universitätsklinikum Carl Gustav Carus Dresden, Technische Universität Dresden, 2019. https://nbn-resolving.org/urn:nbn:de:bsz:14-qucosa2-723869 (accessed January 13, 2021).

[7] A.J. Masino, E.T. Dechene, M.C. Dulik, A. Wilkens, N.B. Spinner, I.D. Krantz, J.W. Pennington, P.N. Robinson, and P.S. White, Clinical phenotype-based gene prioritization: an initial study using semantic similarity and the human phenotype ontology, BMC Bioinformatics. 15 (2014) 248. doi:10.1186/14712105-15-248.

[8] S. Nguengang Wakap, D.M. Lambert, A. Olry, C. Rodwell, C. Gueydan, V. Lanneau, D. Murphy, Y. Le Cam, and A. Rath, Estimating cumulative point prevalence of rare diseases: analysis of the Orphanet database, Eur. J. Hum. Genet. 28 (2020) 165-173. doi:10.1038/s41431-019-0508-0.

[9] ORPHANET and EURODIS, Rare Diseases in Numbers - Preliminary report from an on going bibliographic studyinitiated by Eurordis in partnership with Orphanet, (2005). https://www.orpha.net/actor/Orphanews/2005/doc/Rare_Diseases_in_Numbers.pdf (accessed March 25, 2021).

[10] A. Rath, A. Olry, F. Dhombres, M.M. Brandt, B. Urbero, and S. Ayme, Representation of rare diseases in health information systems: The orphanet approach to serve a wide range of end users, Hum. Mutat. 33 (2012) 803-808. doi:10.1002/humu.22078.

[11] J. Schmitt, T. Lange, C. Forkert, M. Rößler, F. Walther, A. Knapp, J. Karschau, M. Gruhl, M. Kümmel, R. Gebler, M. Löwe, M. Sedlmayr, A. Mogwitz, and C. Kleber, Das DISPENSE-Tool: Dresdner Informations- und Prognosetool für Bettenauslastung in Sachsen, in: German Medical Science GMS Publishing House, 2020. doi:10.3205/20dkvf048.

[12] R. Shailam, A. Botwin, M. Stout, and M.S. Gee, Real-Time Electronic Dashboard Technology and Its Use to Improve Pediatric Radiology Workflow, Curr. Probl. Diagn. Radiol. 47 (2018) 3-5. doi:10.1067/j.cpradiol.2017.03.002.

[13] P.A. Twohig, J.R. Rivington, D. Gunzler, J. Daprano, and D. Margolius, Clinician dashboard views and improvement in preventative health outcome measures: a retrospective analysis, BMC Health Serv. Res. 19 (2019) 475. doi:10.1186/s12913-019-4327-3.

[14] B.A. Wilbanks, and P.A. Langford, A Review of Dashboards for Data Analytics in Nursing, CIN Comput. Inform. Nurs. 32 (2014) 545-549. doi:10.1097/CIN.0000000000000106. 